\title{
ORIGINAL ARTICLE \\ Exploratory analysis of factors associated with venous thromboembolism in Victorian acute traumatic spinal cord-injured patients 2010-2013
}

\author{
R Clements ${ }^{1}$, L Churilov ${ }^{2}$, ALA Wahab ${ }^{1}$ and LC Ng${ }^{1}$
}

Study design: A retrospective medical records audit.

Objectives: To investigate the presence of venous thromboembolism (VTE) events following acute traumatic spinal cord injury (SCI) and the association between VTE events and a number of postulated risk factors.

Setting: The state-wide SCI service in Victoria Australia (Victorian Spinal Cord Service) located at Austin Hospital Melbourne Australia. Methods: A retrospective electronic medical records file audit was performed of all patients admitted to VSCS between 2010 and 2013 with an acute traumatic SCI. The outcome measure was the presence of VTE (deep venous thrombosis (DVT), pulmonary embolism (PE) or both). Data were also collected on a variety of established and postulated risk factors for VTE post SCl.

Results: VTE events occurred in $21.2 \%$ of acute $\mathrm{SCl}$ patients during the hospitalisation of the patient. Statistically significant associations were found between the presence of VTE events and increased weight, male sex, completeness of motor paralysis, length of stay (LOS), associated pelvic or lower limb fracture and delayed admission to the state-wide spinal cord service.

Conclusion: Further studies are warranted to investigate whether in other $\mathrm{SCl}$ centres the risk of VTE in acute SCl patients is similarly associated with the risk factors identified in our study. A study exploring whether giving acute $\mathrm{SCl}$ patients of heavier weight a larger dose of chemical thromboprophylaxis is safe and efficacious is also warranted.

Spinal Cord (2017) 55, 74-78; doi:10.1038/sc.2016.94; published online 14 June 2016

\section{INTRODUCTION}

Venous thromboembolism (VTE) is a significant cause of morbidity and mortality in the acute phase of spinal cord injury (SCI), and without prophylaxis the incidence of deep venous thrombosis (DVT) in acute SCI may be as high as $100 \% .{ }^{1}$ In the first month after acute SCI, these patients have a 500 -fold increased rate of pulmonary embolism (PE)-related death compared with age and gender-matched controls without an acute SCI. ${ }^{2}$ Extensive literature has explored recommended regimes for VTE prophylaxis in acute SCI, as well as risk factors for VTE in acute SCI. ${ }^{1,3-11}$

The Victorian Spinal Cord Service (VSCS) is one of the six spinal specialist services in Australia. It provides acute management and rehabilitation for people who sustain traumatic and some non-traumatic SCIs from Victoria, Tasmania and the Riverina of New South Wales (NSW). The service admits approximately 80 new acute traumatic SCI patients per year and has the capacity to provide lifelong follow-up to them. VTE prevention policy for acute SCI patients in our service includes $40 \mathrm{mg}$ of enoxaparin daily from immediately after the injury if there are no contraindications, graduated compression stockings (patients usually arrive from the referring hospital or Emergency Department already wearing these) and during the complete bed rest use of sequential calf compression devices. Enoxaparin $40 \mathrm{mg}$ daily and compression stockings are given for 3 months post the injury.
A detailed analysis of VTE events occurred with a view to ensure that VTE incidence is as low as possible and prophylaxis is optimised. We hypothesised that there would be an association between the incidence of VTE and various demographic and process-of-care characteristics including weight, operative vs non-operative management, time from injury to commencement of chemoprophylaxis, coexistent lower limb or pelvic fractures and completeness of paralysis. We also hypothesised that there would be an association between sustaining a VTE event and patient's overall length of stay (LOS).

\section{MATERIALS AND METHODS}

\section{Data acquisition}

A retrospective electronic medical records file audit was performed of all patients admitted to VSCS between 2010 and 2013 with an acute traumatic SCI. Approval was obtained by Austin Health Human Research Ethics Committee.

Outcome measures collected were episodes of radiologically confirmed VTE events. Also collected were data on a number of variables that are established and postulated risk factors for VTE post SCI. These included age, weight, sex, details of time of injury, admission American Spinal Injury Association (AIS) impairment score (an assessment of the level and completeness of the SCI), associated pelvic or lower limb fracture, time in days post injury until admission to VSCS, whether operative treatment occurred for the traumatic spinal injury, delay in days between SCI and commencement of VTE chemoprophylaxis, time post SCI of diagnosed VTE event and a detailed profile of methods and timing of the VTE prophylaxis measures used and LOS.

${ }^{1}$ Victorian Spinal Cord Service Austin Health, Heidelberg, Victoria, Australia and ${ }^{2}$ Statistics and Decision Analysis Academic Platform, The Florey Institute of Neuroscience and Mental Health, RMIT University, Melbourne, Victoria, Australia

Correspondence: Dr R Clements, Victorian Spinal Cord Service Austin Health, Austin Hospital, 145 Studley Road, Heidelberg, Victoria 3084, Australia.

E-mail: richard.clements@austin.org.au

Received 22 December 2015; revised 10 April 2016; accepted 17 May 2016; published online 14 June 2016 


\section{Main measures}

Completeness of paralysis was defined by the AIS score. Patients with AIS A or $\mathrm{B}$ initial classifications were regarded as 'motor complete' and AIS C, D or E as motor incomplete. VTE events (DVT or PE) were not routinely screened for. Rather radiological tests were conducted to confirm or refute a clinically suspected VTE event. These radiological tests were venous Doppler ultrasound for suspected DVT and nuclear medicine ventilation perfusion (VQ) scan or Computerised Tomography Pulmonary Angiogram (CTPA) for suspected PE.

LOS referred to the duration in days the patient was a hospital inpatient in the Victorian Spinal Cord Service after their acute injury and was the sum of the acute VSCS admission and the VSCS inpatient rehabilitation admission (if the patient was admitted for inpatient rehabilitation).

\section{Statistical analysis}

The association between various patients' characteristics and the presence or absence of VTE was investigated using Fisher's exact test for baseline characteristics measured on categorical scales and the Wilcoxon-MannWhitney test for continuous characteristics. Demographic and process-of-care characteristics that were associated with the VTE outcome in a statistically significant manner were subsequently included in a multivariable logistic regression model for the purposes of adjusted analysis. The effect sizes are reported as odds ratios (ORs) with corresponding 95\% confidence intervals (95\% CIs). Standard diagnostics of collinearity and model fit was performed. Identical analyses were also conducted for DVT and PE outcomes separately.

The association between the absence or presence of VTE and hospital LOS was investigated using the Wilcoxon-Mann-Whitney rank-sum test.

For all analyses, the values of $P<0.05$ were regarded as indicative of statistical significance, and no adjustments for multiplicity of comparisons were undertaken because of the exploratory nature of this study. The analyses were conducted using Stata IC v13 statistical software (StataCorp, College Station, TX, USA).

\section{RESULTS}

There were 222 patients. As regards the aetiology of their acute SCI, 81 (36\%) were due to falls, 76 (34\%) due to motor vehicle accidents, $14(6 \%)$ due to pushbike accidents, $14(6 \%)$ due to diving or surfing accidents, $10(5 \%)$ due to sporting accidents (3 rugby, 3 Australian Rules football, 2 trampolining, 2 snowboarding), 9 (4\%) due to horse riding accidents, $4(2 \%)$ due to logging or tree falling accidents and 1 each due to gunshot, stabbing, electrocution and indeterminate aetiology. Of the 222, 48 (21.6\%) were female and 174 (78.4\%) were male. All were admitted to VSCS with acute traumatic SCI in the period 2010-2013. Of these 222, $47(21.2 \%)$ were diagnosed with a VTE event. Of the 175 patients who were not diagnosed with a VTE event, $107(61.1 \%)$ did not have any radiological investigations to exclude VTE, and 68 (38.9\%) had at least one negative radiological investigation looking for a VTE event.

Of the 47 who were diagnosed with a VTE event, $30(63.8 \%)$ had a DVT, $25(53.2 \%)$ had a PE and $8(17 \%)$ had both. Forty-three $(91.5 \%)$ were male and four $(8.5 \%)$ were female. In all, $41(87.2 \%)$ had the VTE event diagnosed in the first 3 months post injury, $2(4.3 \%)$ between 3 and 4 months post injury, 3 (6.4\%) between 4 and 5 months post injury and $1(2.1 \%)$ between 5 and 6 months post injury. Table 1 demonstrates the distribution of the various characteristics in the patient populations. Table 2 lists those variables with and without a statistically significant relationship to the key outcomes of VTE, DVT and PE.

\section{DVT outcome}

Thirty of the two hundred twenty-two patients (13.5\%) sustained a DVT. In all, 27 of $30(90 \%)$ occurred in the first 3 months after the

Table 1 Patient variables

\begin{tabular}{|c|c|c|c|c|c|c|c|}
\hline Variable & Total $(\mathrm{N}=222)$ & $\begin{array}{c}\text { VTE positive } \\
(\mathrm{N}=47)\end{array}$ & $\begin{array}{c}\text { VTE negative } \\
(\mathrm{N}=175)\end{array}$ & $\begin{array}{c}\text { DVT positive } \\
(\mathrm{N}=30)\end{array}$ & $\begin{array}{c}\text { DVT negative } \\
\qquad(\mathrm{N}=192)\end{array}$ & $P E$ positive $(\mathrm{N}=25)$ & $\begin{array}{c}\text { PE negative } \\
(\mathrm{N}=197)\end{array}$ \\
\hline Age (median years, IQR) & $44(25-61)$ & $44(31-56)$ & $43(25-62)$ & $47.5(31-56)$ & $43(25-62)$ & $44(31-54)$ & $44(25-62)$ \\
\hline \multicolumn{8}{|l|}{ Gender } \\
\hline Male & $174(78)$ & $43(91)$ & $131(75)$ & $27(90)$ & $147(77)$ & $24(96)$ & $150(76)$ \\
\hline Female & $48(22)$ & $4(9)$ & $44(25)$ & $3(10)$ & $45(23)$ & $1(4)$ & $47(24)$ \\
\hline \multicolumn{8}{|l|}{ Lesion level } \\
\hline Tetraplegia & $139(63)$ & $25(53)$ & $114(65)$ & $13(43)$ & $126(66)$ & $13(52)$ & $126(64)$ \\
\hline Paraplegia & $83(37)$ & $22(47)$ & $61(35)$ & $17(57)$ & $66(34)$ & $12(48)$ & $71(36)$ \\
\hline \multicolumn{8}{|l|}{ Motor completeness } \\
\hline AIS A/B & $139(63)$ & $35(74)$ & $104(59)$ & $20(67)$ & $119(62)$ & $21(84)$ & $118(60)$ \\
\hline AIS C/D & $83(37)$ & $12(26)$ & $71(41)$ & $10(33)$ & $73(38)$ & $4(16)$ & $79(40)$ \\
\hline \multicolumn{8}{|l|}{ Operative management acutely } \\
\hline Operated & $184(83)$ & $38(81)$ & $146(83)$ & $26(87)$ & $152(82)$ & $19(76)$ & $165(84)$ \\
\hline Not operated & $38(17)$ & $9(19)$ & $29(17)$ & $4(13)$ & $34(18)$ & $6(24)$ & $32(16)$ \\
\hline Weight (median kg, IQR) & $75.5(68-87)$ & $82(75-93)$ & $75(67-85)$ & 80.5 (75-97) & $75(68-85)$ & $84(77-93)$ & $75(67-85)$ \\
\hline \multicolumn{8}{|c|}{ Associated pelvic or lower limb fracture } \\
\hline Present & $17(8)$ & $7(15)$ & $10(6)$ & $6(20)$ & $11(6)$ & $3(12)$ & $14(7)$ \\
\hline Absent & $205(92)$ & $40(85)$ & $165(94)$ & $24(80)$ & $181(94)$ & $22(88)$ & $183(93)$ \\
\hline $\begin{array}{l}\text { Time from injury to admission } \\
\text { to State-wide Service, days } \\
\text { (median, IQR) }\end{array}$ & $1(1-8)$ & $2(1-17)$ & $1(1-7)$ & $3.5(1-17)$ & $1(1-7)$ & $2(1-16)$ & $1(1-8)$ \\
\hline Delay from injury to com- & $3(2-6)$ & $3(2-6)$ & $3(2-5)$ & $4.5(2-6)$ & $3(2-5)$ & $3(2-7)$ & $3(2-5)$ \\
\hline
\end{tabular}

mence anti-coagualtion che-

moprophylaxis, days (median,

IQR)

LOS, days (median, IQR) 
Table 2 Relationship between patient variables and incidence of DVT, PE and VTE incidence

\begin{tabular}{|c|c|c|c|c|c|c|c|c|c|}
\hline \multirow[t]{2}{*}{ Variable } & \multicolumn{3}{|c|}{$D V T$} & \multicolumn{3}{|c|}{$P E$} & \multicolumn{3}{|c|}{ VTE } \\
\hline & $\begin{array}{l}\text { Univariate } \\
\text { P-value }\end{array}$ & $\begin{array}{l}\text { Multivariable } \\
\text { OR }(95 \% \mathrm{Cl})\end{array}$ & $\mathrm{P}$-value & $\begin{array}{l}\text { Univariate } \\
\text { P-value }\end{array}$ & $\begin{array}{l}\text { Multivariable } \\
\text { OR }(95 \% \mathrm{Cl})\end{array}$ & $\mathrm{P}$-value ${ }^{\mathrm{b}}$ & $\begin{array}{l}\text { Univariate } \\
\text { P-value }\end{array}$ & $\begin{array}{l}\text { Multivariable } \\
\text { OR }(95 \% \mathrm{Cl})\end{array}$ & $\mathrm{P}$-value $\mathrm{b}^{\mathrm{b}}$ \\
\hline Age (per year) & 0.73 & & & 0.88 & & & 0.89 & & \\
\hline Male sex & 0.15 & & & 0.02 & $\begin{array}{c}5.02 \\
(0.64-39.5)\end{array}$ & 0.13 & 0.02 & $\begin{array}{c}2.47 \\
(0.78-7.89)\end{array}$ & 0.13 \\
\hline Weight (per kg increase) & 0.03 & $\begin{array}{c}1.03 \\
(1.00-1.05)\end{array}$ & 0.01 & 0.0007 & $\begin{array}{c}1.03 \\
(1.00-1.06)\end{array}$ & 0.03 & 0.0007 & $\begin{array}{c}1.03 \\
(1.01-1.05)\end{array}$ & 0.01 \\
\hline Motor paralysis complete & 0.68 & & & 0.03 & $\begin{array}{c}3.19 \\
(1.03-9.85)\end{array}$ & 0.04 & 0.06 & $\begin{array}{c}1.78 \\
(0.81-3.91)\end{array}$ & 0.15 \\
\hline Paraplegic vs tetraplegic & 0.03 & $\begin{array}{c}0.47 \\
(0.20-1.08)\end{array}$ & 0.08 & 0.27 & & & 0.17 & & \\
\hline Operative management of acute injury & 0.79 & & & 0.4 & & & 0.67 & & \\
\hline $\begin{array}{l}\text { Time in days between injury and commencement of } \\
\text { anticoagulant chemoprophylaxis (per day increase) }\end{array}$ & 0.23 & & & 0.82 & & & 0.39 & & \\
\hline $\begin{array}{l}\text { Time in days between injury and commencement of } \\
\text { anticoagulant chemoprophylaxis (per day increase) }\end{array}$ & 0.03 & $\begin{array}{c}1.02 \\
(1.00-1.05)\end{array}$ & 0.03 & 0.16 & & & 0.04 & $\begin{array}{c}1.03 \\
(1.00-1.05)\end{array}$ & 0.02 \\
\hline Associated lower limb or pelvic fracture & 0.02 & $\begin{array}{c}2.45 \\
(0.96-6.26)\end{array}$ & 0.06 & 0.42 & & & 0.06 & $\begin{array}{c}2.25 \\
(0.87-5.81)\end{array}$ & 0.09 \\
\hline Length of stay (per day increase) & 0.02 & & & 0.002 & & & $<0.0001$ & & \\
\hline
\end{tabular}

Abbreviations: AIS, American Spinal Injury Association; CI, confidence interval; DVT, deep venous thrombosis; OR, odds ratio; PE, pulmonary embolism; VTE, venous thromboembolism.

a $P$-value for the continuous Wilcoxon-Mann-Whitney rank-sum test for Fisher's exact test.

b $P$-value calculated using multiple logistic regression.

accident, 1 in the fourth month after the accident (3.3\%) and 2 in the fifth month after the injury $(6.7 \%)$.

In the univariate analysis, the presence of DVT was statistically significantly associated with patients' weight (Wilcoxon-MannWhitney $P=0.03)$, lower limb or pelvic fracture $(P=0.02)$, paraplegia vs quadriplegia $(P=0.03)$, time in days between injury and admission to the VSCS (Wilcoxon-Mann-Whitney $P=0.03$ ) and LOS (Wilcoxon-Mann-Whitney $P=0.02$ ). No statistically significant association between DVT patients' sex, age, completeness of paralysis, operative vs non-operative management of the acute injury and time in days between injury and commencement of anticoagulation was identified.

A multivariable logistic regression model for DVT was performed with the four factors that in univariate analysis had a statistically significant association with sustaining a DVT being weight, paraplegia vs quadriplegia, presence of an associated pelvic or lower limb fracture and time from injury to admission to the VSCS. On this multivariable analysis, weight (per 1-kg increase in weight, the odds of developing a DVT are increased by $3 \%$ : OR $1.03,95 \%$ CI $1.00-1.05, P=0.01)$ and time from injury to admission to VSCS (per extra 1-day delay the odds of developing DVT are increased by $2 \%$ : OR 1.02, 95\% CI 1.00-1.05, $P=0.03)$ retained statistical significance. Paraplegia vs quadriplegia $(P=0.08)$ and presence of pelvic or lower limb fracture $(P=0.06)$ did not retain statistical significance.

\section{PE outcome}

PE occurred in 25 of the 222 (11.3\%) of patients. In all, 22 of the PEs occurred in the first 3 months post injury with 1 between 3 and 4 months post injury, 1 between 4 and 5 months post injury and 1 between 5 and 6 months post injury.

In the univariate analysis, the presence of $\mathrm{PE}$ was statistically significantly associated with patients' weight (Wilcoxon-MannWhitney $P=0.0007)$, male sex $(P=0.02)$, motor paralysis completeness $(P=0.03)$ and LOS (Wilcoxon-Mann-Whitney $P=0.002)$. No statistically significant associations between $P E$ and patients' age, operative vs non-operative management of the acute injury, time in days between injury and commencement of anticoagulation, paraplegia vs quadriplegia, time between injury and admission to VSCS and associated lower limb or pelvic fracture were identified.

A multivariable logistic regression model for PE was performed with the three factors that in univariate analysis had a statistically significant association with sustaining a PE being weight, male sex and completeness of motor paralysis. In this multivariate analysis, motor paralysis completeness (OR 3.19, 95\% CI 1.03-9.85, $P=0.04$ ) and weight (per 1-kg increase in weight, the odds of developing a PE are increased by $3 \%$ : OR $1.03,95 \%$ CI $1.00-1.06, P=0.03)$ retained statistical significance, whereas male sex did not $(P=0.13)$.

\section{VTE outcome}

VTE event was defined as either PE or DVT. In all, 47 of $222(21.2 \%)$ patients sustained a VTE. In the univariate analysis, the presence of VTE was statistically significantly associated with patients' weight (Wilcoxon-Mann-Whitney $P=0.0007)$, male sex $(P=0.02)$, time from injury to admission to VSCS (Wilcoxon-Mann-Whitney $P=0.04$ ) and LOS (Wilcoxon-Mann-Whitney $P<0.0001$ ). No statistically significant association between VTE and lower limb/pelvic fracture $(P=0.06)$ and motor paralysis completeness $(P=0.06)$ was identified, but the relationship was very close to being statistically significant. No statistically significant association between VTE and patients' age, operative vs non-operative management of the acute injury, time in days between injury and commencement of anticoagulation, paraplegia vs quadriplegia, motor paralysis completeness and associated lower limb or pelvic fracture was identified. 
A multivariable logistic regression model for VTE was performed with the three factors that in univariate analysis had a statistically significant association with sustaining a VTE-namely, male sex, weight and time in days from injury and admission to the VSCS - and the two factors that in univariate analysis had an almost statistically significant association with sustaining a VTE—namely, coexistent lower limb/pelvic fracture and completeness of motor paralysis.

In this multivariate analysis, time from injury to admission to VSCS (per extra 1-day delay, the odds of developing VTE are increased by 3\%: OR 1.03, 95\% CI 1.00-1.05, P=0.02) and weight (per $1-\mathrm{kg}$ increase in weight, the odds of developing a VTE are increased by $3 \%$ : OR $1.03,95 \%$ CI 1.01-1.05, $P=0.01)$ retained statistical significance, whereas male sex $(P=0.13)$, coexistent lower limb/pelvic fracture $(P=0.09)$ and completeness of motor paralysis $(P=0.15)$ did not.

\section{DISCUSSION}

In our study, the incidence of VTE in acute SCI patients was $21.2 \%$ during the initial hospitalisation of the patient (including the inpatient rehabilitation phase). Previous studies have noted a widely varying incidence and prevalence of DVT and PE in acute SCI (ranging from 9 to $100 \%){ }^{10}$ The explanation for this large variation likely is the unreliability of clinical diagnosis of VTE combined with variability of diagnostic regimes (routine screening vs testing only upon clinical suspicion, VQ scan vs CTPA, D Dimer use or not).

Our study found increased weight to be statistically significantly associated with DVT, PE and VTE in acute SCI. Our unit prophylaxis policy includes daily $40 \mathrm{mg}$ enoxaparin regardless of the patient's weight. Early proponents of low molecular weight heparin argued that one of its advantages was that dose adjustment was not necessary. ${ }^{10,12}$ However, for the prophylactic use of enoxaparin in SCI, the optimal dose is not yet certain. ${ }^{10}$ One previous study of 129 patients by Hebbeler et al. ${ }^{13}$ found no difference in VTE events or bleeding complications in acute SCI patients given $40 \mathrm{mg}$ daily vs $30 \mathrm{mg}$ twice-daily. For enoxaparin treatment of DVT or PE the dose is weight adjusted ( $1 \mathrm{mg} \mathrm{kg}^{-1}$ bd a common recommendation). However, no VTE in acute SCI study has varied the prophylactic enoxaparin dose according to weight. Given in our cohort that all patients received the 40-mg dose regardless of weight and heavier patients had a greater VTE risk such a study is worthy of consideration.

The statistically significant association between male sex and PE and VTE in acute SCI patients has been noted in other studies. ${ }^{5,11}$ Both Jones et al. ${ }^{5}$ and Selassie et al. ${ }^{11}$ found statistically significant association between VTE and male sex post acute SCI, although not all studies have found this. The male preponderance is not well understood. It did not retain statistically significantly association on multivariable analysis.

The statistically significant association between completeness of motor paralysis and PE incidence (and almost statistically significantly association between VTE and completeness of motor paralysis) has also been demonstrated in other studies ${ }^{5,11}$ and intuitively is explicable by venous stasis being greater in the more completely paralysed muscle.

The statistically significant association between associated lower limb/pelvic fracture and DVT is consistent with established knowledge of the risk of VTE from lower limb and pelvic fractures due to the potential for endothelial trauma and venous stasis from immobility from these injuries.

There was a statistically significant association between both DVT and VTE with increasing time between injury and admission to the acute state-wide SCI service. This was studied because of an anecdotal observation from the staff in the state-wide SCI service that patients who spent a longer time in a different acute facility before admission to our state-wide SCI service often commenced DVT chemoprophylaxis later after their acute injury and had a greater incidence of VTE. For methodological reasons, this was difficult to prove as it was not always possible to ascertain with certainty the exact time of chemoprophylaxis commencement at the referring hospital. Physicians in the state-wide SCI service, keenly aware of the significant incidence of PE in their patients, when assessing the risks and benefits of anticoagulant chemoprophylaxis prescribe it unless there are robust contraindications. It is commenced within $24-48 \mathrm{~h}$ of major spinal surgery, and a presence of intramedullary blood on magnetic resonance imaging in a complete patient is not viewed as a contra-indication. Our impression is that in acute referring hospitals (for example regional hospitals, or metropolitan major trauma centres), chemoprophylaxis is commonly commenced later (at times not at all in the case of demonstrable intracord blood on magnetic resonance imaging).

One of the reasons patients may take a longer time to be transferred to the state-wide SCI service is the presence of extensive other major traumatic injuries. In those cases, there may be valid contraindications to early anticoagulant chemoprophylaxis (major vascular injury, frequent operative procedures and so on).

The strong association between an acute SCI patient sustaining a VTE event and a longer LOS has been noted by previous researchers, ${ }^{11}$ although Selassie et al. measured LOS as the duration of acute hospitalisation, whereas this study counted the inpatient rehabilitation phase too. Whether it is the VTE event alone that prolongs the LOS is doubtful. Other factors that make VTE more likely (such as severity of motor paralysis completeness) also likely prolong LOS. There are some important limitations in our study. This was a retrospective audit as opposed to a prospective study. This meant that radiological assessment was not carried out in 107 patients (in whom there was no clinical suspicion of a VTE event).

Given the notorious difficulty in excluding VTE purely by clinical assessment inevitably, this means that the true rate of DVT, PE and VTE have been underestimated in this study. Also, given radiological tests were only conducted upon VTE clinical suspicion, the timing post injury of development of VTE will be less accurate than if screening investigations at multiple time points had occurred. A prospective study in which all patients received screening diagnostic imaging at set times would give a more accurate incidence of VTE and a more accurate estimate of their time of development. It is noted that $87.2 \%$ of the VTE events that occurred took place in the first 3 months post injury with the remaining $12.8 \%$ occurring between 3 and 6 months post injury (thus $2.7 \%$ of our patients suffered a VTE event between 3 and 6 months post acute SCI despite receiving 3 months of VTE prophylaxis). This raises the question of how long VTE prophylaxis should continue. In our unit, 3 months is the routine duration. Guidelines in the literature have recommended that at least 3 months of VTE prophylaxis be given following the acute SCI. ${ }^{7}$ Further studies are warranted to assess the risks vs benefits of extending further the duration of VTE prophylaxis in some or all of acute SCI patients.

\section{CONCLUSION}

Our study displayed statistically significant associations between VTE, $\mathrm{PE}$ and DVT and a number of predictable risk factors including increased weight (VTE, PE and DVT), completeness of motor paralysis (PE), male sex (PE and VTE) and associated lower limb or pelvic fracture (DVT). Increased weight was the only risk factor in our study with statistically significant associations in both univariate and 
multivariable analyses with all three outcomes (PE, DVT and VTE). In our state-wide SCI service, practise has been to provide the same VTE prevention regime (provided there are no contraindications) regardless of the presence or absence of risk factors that make VTE events in these patients even more likely than the acute SCI does alone (such as increased weight, completeness of motor paralysis, or associated pelvic or lower limb fracture). Further study is warranted, first to determine whether, in other SCI centres, the same factors we have found further increase VTE risk in acute spinal cord-injured patients. Second, a study is warranted of whether increased dose of chemical thromboprophylaxis in acute spinal cord-injured patients with heavier weight can safely reduce the increased risk of VTE events in these patients without conferring an unacceptable increased risk of bleeding.

\section{DATA ARCHIVING}

There were no data to deposit.

\section{CONFLICT OF INTEREST}

The authors declare no conflict of interest.

1 Christie S, Thibault-Halman G, Casha S. Acute pharmacological DVT prophylaxis after spinal cord injury. [Review]. J Neurotrauma 2011; 28: 1509-1514.

2 DeVivo MJ, Kartus PL, Stover SL, Rutt RD, Fine PR. Cause of death for patients with spinal cord injuries. Arch Intern Med 1989; 149: 1761-1766.
3 Dhall SS, Hadley MN, Aarabi B, Gelb DE, Hurlbert RJ, Rozzelle CJ et al. Deep venous thrombosis and thromboembolism in patients with cervical spinal cord injuries. [Review]. Neurosurgery 2013; 72: 244-254.

4 Chung SB, Lee SH, Kim ES, Eoh W. Incidence of deep vein thrombosis after spinal cord injury: a prospective study in 37 consecutive patients with traumatic or nontraumatic spinal cord injury treated by mechanical prophylaxis. J Trauma 2011; 71: 867-870 discussion 870-1.

5 Jones T, Ugalde V, Franks $\mathrm{P}$, Zhou H, White RH. Venous thromboembolism after spinal cord injury: incidence, time course, and associated risk factors in 16,240 adults and children. Arch Phys Med Rehabil 2005; 86: 2240-2247.

6 Green D, Hartwig D, Chen D, Soltysik RC, Yarnold PR. Spinal cord injury risk assessment for thromboembolism (SPIRATE study). Am J Phys Med Rehabil 2003; 82: 950-956.

7 Geerts WH, Bergqvist D, Pineo GF, Heit JA, Samama CM, Lassen MR et al. Prevention of venous thromboembolism: American College of Chest Physicians. Evidence Based Clinical Practice Guidelines (8th Edition). Chest 2008; 133: 381S-453S.

8 Bullano MF, Willey V, Hauch O, Wygant G, Spyropoulos AC, Hoffman L. Longitudinal evaluation of health plan cost per venous thromboembolism or bleed event in patients with a prior venous thromboembolism event during hospitalization. J Manag Care Pharm 2005; 11: 663-673.

9 Furlan JC, Fehlings MG. Role of screening tests for deep venous thrombosis in asymptomatic adults with acute spinal cord injury: an evidence-based analysis. [Review] [72 refs]. Spine 2007; 32: 1908-1916.

10 Teasell RW, Hsieh JT, Aubut JL, Eng JJ, Krassioukov A, Tu L. Spinal Cord Injury Rehabilitation Evidence Review Research Team Venous thromboembolism after spinal cord injury. Arch Phys Med Rehabil 2009; 90: 232-245.

11 Selassie AW, Varma A, Saunders LL. Current trends in venous thromboembolism among persons hospitalized with acute traumatic spinal cord injury: does early access to rehabilitation matter? Arch Phys Med Rehabil 2011; 92: 1534-1541.

12 Rydberg EJ, Westfall JM, Nicholas RA. Low-molecular-weight heparin in preventing and treating DVT. Am Fam Physician 1999; 59: 1607-1612.

13 Hebbeler SL, Marciniak CM, Crandall S, Chen D, Nussbaum S, Mendelewski S. Daily vs twice daily enoxaparin in the prevention of venous thromboembolic disorders during rehabilitation following acute spinal cord injury. J Spinal Cord Med 2004; 27: 236-240. 